\title{
Anticodon loop mutations perturb reading frame maintenance by the E site tRNA
}

\author{
CHRISTINA L. SANDERS, KRISTIN J. LOHR, HOLLY L. GAMBILL, RYAN B. CURRAN, and JAMES F. CURRAN
}

Department of Biology, Wake Forest University, Winston-Salem, North Carolina 27106, USA

\begin{abstract}
The ribosomal E site helps hold the reading frame. Certain tRNA mutations affect translation, and anticodon loop mutations can be especially detrimental. We studied the effects of mutations saturating the anticodon loop of the amber suppressor tRNA, Su7, on the ability to help hold the reading frame when in the E site. We also tested three mutations in the anticodon stem, as well as a mutation in the D stem (the "Hirsh" mutation). We used the Escherichia coli RF2 programmed frameshift site to monitor frame maintenance. Most anticodon loop mutations increase frameshifting, possibly by decreasing codon:anticodon stability. However, it is likely that the A site is more sensitive to anticodon loop structure than is the E site. Unexpectedly, the Hirsh mutation also increases frameshifting from the $E$ site. Other work shows that mutation may increase the ability of tRNA to react in the A site, possibly by facilitating conformational changes required for aminoacyl-tRNA selection. We suggest that this property may decrease its ability to bind to the E site. Finally, the absence of the $\mathrm{ms}^{2}{ }^{2} \mathrm{o}^{6} \mathrm{~A}$ nucleoside modifications at A37 does not decrease the ability of tRNA to help hold the reading frame from the $E$ site. This was also unexpected because the absence of these modifications affects translational properties of tRNA in A and P sites. The absence of a negative effect in the E site further highlights the differences among the substrate requirements of the ribosomal coding sites.
\end{abstract}

Keywords: decoding; frameshift; ribosome; transfer RNA; translational properties

\section{INTRODUCTION}

Maintenance of the reading frame is a critical function in translation, and although the detailed molecular mechanism is not fully clear, it must depend tRNA:message interaction. The $\mathrm{P}$ site tRNA is well known to play a critical role (see, e.g., Baranov et al. 2004), and recent evidence strongly suggests that the E site tRNA helps hold the reading frame. For example, Marquez et al. (2004) show that tRNA cognate to the codon in the E site inhibits the RF2 programmed frameshift in vitro. And we show that for 29 different RF2 alleles in vivo, frameshift frequency is quantitatively correlated with the stabilities of codon:anticodon duplexes in the E site, such that weak pairing allows for more frameshifting (Sanders and Curran 2007). Those data clearly implicate codon:anticodon interaction in the $\mathrm{E}$ site as an important contributor to reading frame maintenance. In addition, an rRNA mutation that decreases tRNA binding in the $\mathrm{E}$ site is also associated with slightly increased

Reprint requests to: James F. Curran, Department of Biology, Wake Forest University, 1834 Wake Forest Road, Winston-Salem, NC 27106, USA; e-mail: curran@wfu.edu; fax: (336) 758-6008.

Article published online ahead of print. Article and publication date are at http://www.rnajournal.org/cgi/doi/10.1261/rna.1170008. levels of frameshifting (Sergiev et al. 2005). Together, these studies argue that the E site duplex inhibits frameshifting, and suggest that factors that promote duplex dissociation in the E site may increase frameshifting.

It is well known that anticodon stem-loop nucleotides outside of the anticodon itself affect the decoding efficiency of tRNA. Yarus (1982) pointed out that the identities of nucleotides in the anticodon stem-loop are strongly correlated with nucleotide 36 , the anticodon nucleotide that reads the first codon nucleotide. He suggested that the anticodon stem-loop acts as an "extended anticodon," as if neighboring nucleotides help position the anticodon for optimal interaction with the codon. A host of data supports this model. For example, he also noted that nonsense suppressors derived from tRNAs that normally read codons starting with " $U$ " and having, therefore, extended anticodon stem-loop sequences that may be appropriate for nonsense codons, are generally more efficient than suppressors derived from tRNAs that read codons starting with other nucleotides (Yarus 1982). Also consistent with this hypothesis, mutations in the anticodon loop of nonsense suppressors that bring the extended anticodon sequence closer to (Raftery and Yarus 1987) or farther from the consensus (Yarus et al. 1986) have predictable effects on translational efficiency. 
An extensive mutational analysis of the anticodon stemloop of the amber suppressor derived from tRNA ${ }^{\text {Trp }}$ (called Su7 below) shows that virtually all changes in the anticodon loop decrease suppression efficiency (Yarus et al. 1986). Although some of the decreases were attributed to effects on tRNA concentration and aminoacylation, it is clear that most changes in the anticodon loop also affect translational efficiency. Importantly for the current paper, these variants span a wide range of translational activities, and we hypothesize that decreases in translational efficiency in the ribosomal A site correlate with decreases in the ability to hold the reading frame from the $\mathrm{E}$ site.

Nucleotide modifications in the anticodon loop contribute to decoding efficiency (for review, see Björk 1995; Curran 1998). In particular, nucleotide 37, which is immediately adjacent to the anticodon, is often modified, and these modifications are correlated with anticodon nucleotide 36 (Yarus 1982). NMR studies show that modifications at position 37 may facilitate decoding maintaining an open anticodon loop and by strengthening the codon:anticodon duplex through enhanced base stacking (Cabello-Villegas et al. 2002; Stuart et al. 2003; Durant et al. 2005; reviewed by Agris et al. 2007). Moreover, the absence of the $\mathrm{ms}^{2} \mathrm{i}^{6} \mathrm{~A}$ modifications at this position of Escherichia coli tRNA $^{\text {Trp }}$ decreases the solution stability of it when paired to tRNA ${ }_{1}^{\text {Pro }}$ through their complementary anticodons (Houssier and Grosjean 1985). Mutations that block modification can decrease the translational activities of both nonsense suppressors (Bouadloun et al. 1986; Björnsson and Isaksson 1993) and tRNAs with WT anticodons (Ericson and Björk 1986; Li et al. 1997).

The E. coli prfB (RF2) gene contains a well-characterized programmed frameshift site. High-frequency frameshifting requires a slowly translated A site codon (Curran and Yarus 1988), a slippery codon in the P site (Weiss et al. 1990; Curran 1993), a Shine/Dalgarno-like element to stimulate the frameshift (Weiss et al. 1988), and a weakly paired E site codon:anticodon duplex (Marquez et al. 2004; Sanders and Curran 2007). Critically for the current study, those works show that the E site duplex contributes to the rate-limiting step so that changes in duplex stability should directly affect frameshifting (see also the discussion in Liao et al. 2008).

Here we test the effects of various tRNA mutations on reading frame maintenance while the mutant tRNAs reside in the E site of the RF2 programmed frameshift. Mutations that weaken codon:tRNA duplexes are expected to increase frameshifting. We study Yarus's collection of Su7 mutations that saturate the anticodon loop. We also test several mutations in the anticodon stem and a mutation in the tRNA dihydrouridine stem (D stem). We also study the effect of loss of the hypermodified nucleotide at position 37, $\mathrm{ms}^{2}$ io $^{6} \mathrm{~A}$ (2-methylthio-N-6-[cis-hydroxy] isopentenyl adenosine), for its effect on frame maintenance by $\mathrm{E}$ site tRNAs. We discuss possible molecular explanations for our various results.

\section{RESULTS}

\section{Anticodon loop mutations increase the rate of reading frame loss when in the $E$ site}

To determine how mutations affect frame maintenance when TRNA is in the E site, we studied members of the wellcharacterized tRNA mutation set described by Yarus et al. (1986). This mutation collection saturates the anticodon loop of the Su7 "amber" nonsense suppressor (i.e., translates the UAG or amber stop codon), except for the amber anticodon itself. They made mutations pairwise in the lower portion of the anticodon stem to preserve secondary structure. Here, we studied all of the anticodon loop mutants, and one mutant of each of the three base pairs in the distal portion of the anticodon stem. We did not study all of the anticodon stem mutations in the collection because most of them have similar and relatively minor effects on decoding efficiency (Yarus et al. 1986). We also studied a mutation in the D stem (G>A24) that has been shown to increase decoding efficiency (Hirsh 1971; Raftery et al. 1986). The positions of these mutations are shown in Figure 1. Altogether, we studied 18 Su7 alleles.

To test for effects of anticodon stem-loop mutations on framing from the $\mathrm{E}$ site, we constructed two lac $Z$ alleles that have RF2-derived sequences spliced into a polylinker near the $5^{\prime}$ end (see Materials and Methods). We used the RF2 programmed frameshift because its mechanism is well understood. Figure 2 outlines our model for the ribosomal complex just prior to frameshifting, and the legend describes the mechanism of the frameshift. In all of our experiments below, frameshifting occurs at frequencies of

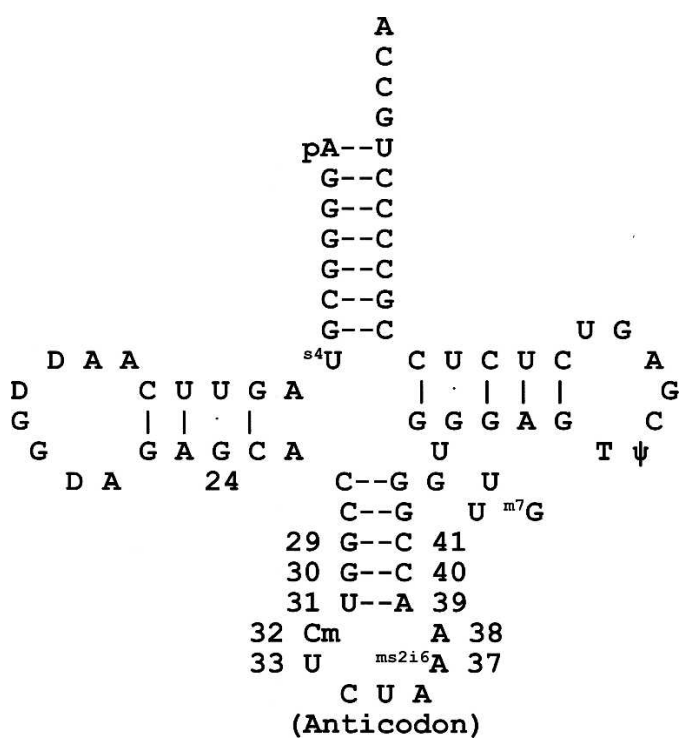

FIGURE 1. The positions of the mutations in Su7. Shown is the cloverleaf structure of Su7. The positions of the mutations studied are marked by nucleotide number. The anticodon is at the bottom and is marked. 


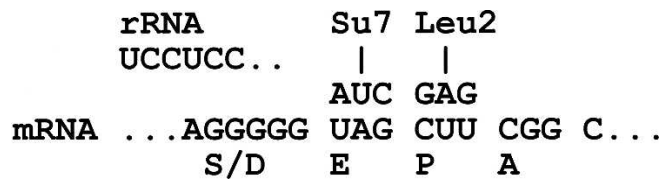

FIGURE 2. The frameshift mechanism of the lacZ/RF2 constructs. Before the frameshift, the mRNA will be paired to an Su7 variant in the ribosomal $\mathrm{E}$ site. The $\mathrm{P}$ site will contain a slippery CUU:tRNA ${ }_{2}^{\mathrm{Leu}}{ }_{2}$ complex, and the A site contains a rare arginine codon that is not paired with any tRNA. Because frameshifting competes with translation of that codon (e.g., Curran and Yarus 1988), the slowly translated rare codon allows for high-efficiency frameshifting (Curran and Yarus 1989). High-frequency frameshifting occurs when the antiShine/Dalgarno of $16 \mathrm{~S}$ rRNA pairs with the Shine/Dalgarno (marked $\mathrm{S} / \mathrm{D}$ in the figure) sequence, the E site tRNA (Su7) dissociates, and tRNA $_{2}^{\text {Leu }}{ }_{2}$ slips rightward onto the overlapping UCC triplet.

$30 \%$ or greater. Because frameshift frequencies at "normal" sites rarely exceed $1 \%$, it is virtually certain that most of our observed high frameshift-dependent activities are due to the RF2 programmed mechanism.

To produce $\beta$-galactosidase in these constructs, the Su7 variant must suppress the UAG codon when it appears in the A site. Then, two elongation cycles later, when the UAG codon:Su7 complex is in the E site, the ribosome either shifts into the " +1 " frame or simply continues in the " 0 " frame. We measured both of these activities using "shift" and "no-shift" reporters. In this study, our empty vector control, which does not encode any suppressor tRNA, gives 5.3 units with the shift reporter and 3 units with the no-shift reporter (Table 1). These results demonstrate that reporter activities of tRNA-expressing clones, which range in the tens to thousands of units, depend almost entirely on Su7 activity.

We measured the $\beta$-galactosidase activities of each of the two reporters in cells that express either the WT tRNA or one of 17 variants. These data are listed in Table 1. Every tRNA/reporter pair was assayed at least eight times. To estimate effects on frameshifting, we calculate frameshift/no-shift ratios. This ratio is an estimate of the relative rates of the frameshift and nonframeshift read-through paths; for example, a ratio of " 1 " means that the two paths have equal rates. The ratio also normalizes for differences in the efficiency with which these mutant tRNAs read the amber codon $5^{\prime}$ of the slippery site. In our earlier paper (Sanders and Curran 2007), because we did not directly measure read-through activity, we could not calculate relative frameshift rates as simple ratios. Instead, we calculated relative frameshift rates from frameshift frequencies relative to a wild-type (WT) lacZ control. However, the relative frameshift rates calculated in the two studies are formally equivalent. To be consistent with our earlier paper, we will use the same abbreviation for relative frameshift rate: FR.

FR varies about fivefold among the tRNA variants, with the WT having the lowest frameshift rate. Altogether, 13 of the 17 mutants frameshift at a significantly higher rate than the WT. These data show that anticodon stem-loop mutations can decrease the ability of tRNA to help hold the reading frame when in the E site.

In Figure 3, FR is plotted versus Yarus's suppression efficiencies for these tRNAs (from Yarus et al. 1986). Suppression efficiency is a measure of the overall ability of the tRNA alleles to function in translation. Although it includes effects that the mutations may have on all aspects of tRNA function (Yarus et al. 1986), it provides a first-order measure of how well the tRNA is selected in the ribosomal A site. The plot shows that there is a general tendency for tRNAs that are less efficient in suppression to also be more frameshift prone. Note that none of the three stem mutations significantly increases FR, but all of the loop mutations except U38 do significantly increase the rate of frame loss. Because stem mutations have little or no effect on
TABLE 1. $\beta$-Galactosidase activities and frameshift frequencies of the Su7 alleles

\begin{tabular}{lccc}
\hline Su7 allele & $\begin{array}{c}\beta \text {-Galactosidase } \\
\text { activity of the } \\
\text { shift construct }\end{array}$ & $\begin{array}{c}\beta \text {-Galactosidase } \\
\text { activity of the } \\
\text { no-shift construct }\end{array}$ & $\begin{array}{c}\text { Frameshift/no-shift } \\
\text { ratio (FR) }\end{array}$ \\
\hline Vector & $5.3 \pm 0.1$ & $3 \pm 0.1$ & - \\
Su7 WT & $2000 \pm 110$ & $4740 \pm 300$ & $0.42 \pm 0.05$ \\
U32 & $1450 \pm 25$ & $2520 \pm 95$ & $0.58 \pm 0.03$ \\
A32 & $270 \pm 25$ & $280 \pm 25$ & $0.96 \pm 0.16$ \\
G32 & $190 \pm 5$ & $320 \pm 10$ & $0.59 \pm 0.03$ \\
C33 & $840 \pm 50$ & $1500 \pm 60$ & $0.56 \pm 0.05$ \\
A33 & $260 \pm 25$ & $250 \pm 25$ & $1.04 \pm 0.19$ \\
G33 & $240 \pm 5$ & $425 \pm 10$ & $0.56 \pm 0.02$ \\
U37 & $45 \pm 5$ & $22 \pm 2$ & $2.04 \pm 0.37$ \\
C37 & $170 \pm 10$ & $220 \pm 20$ & $0.77 \pm 0.1$ \\
G37 & $220 \pm 20$ & $300 \pm 20$ & $0.73 \pm 0.1$ \\
U38 & $770 \pm 15$ & $1680 \pm 130$ & $0.46 \pm 0.04$ \\
C38 & $920 \pm 60$ & $1280 \pm 95$ & $0.72 \pm 0.07$ \\
G38 & 0 & 0 & - \\
G38+U32 & $220 \pm 10$ & $270 \pm 20$ & $0.81 \pm 0.09$ \\
G31+C39 & $2300 \pm 140$ & $4380 \pm 350$ & $0.53 \pm 0.07$ \\
A30+U40 & $2300 \pm 185$ & $4410 \pm 305$ & $0.52 \pm 0.07$ \\
C29+G41 & $1680 \pm 90$ & $3200 \pm 260$ & $0.53 \pm 0.07$ \\
A24 & $2370 \pm 150$ & $3600 \pm 300$ & $0.65 \pm 0.08$ \\
\hline
\end{tabular}

The tRNA alleles are from Yarus et al. (1986). $\beta$-Galactosidase activities for Su7-expressing strains are averages of at least eight assays after subtracting those of the empty vector \pm standard errors of the means. 


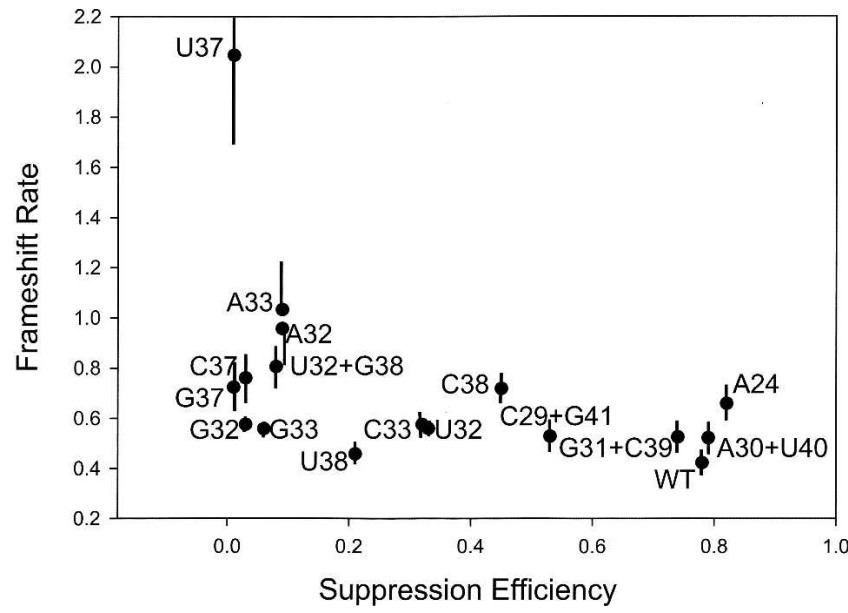

FIGURE 3. Frameshift rate plotted versus suppression efficiency. Suppression efficiencies are from Yarus et al. (1986). Frameshift rates (FR) are from Table 1. The standard errors are marked with lines above and below the average FR values, except that clarity A33 and A32 have just a single side marked.

aminoacyl-tRNA selection while most loop mutations decrease it (Yarus et al. 1986), these results are consistent with the general observation that weak suppressors are more prone to relinquish the frame when in the $\mathrm{E}$ site. However, it is also clear that for most mutations the effects on FR are rather small compared to the effects that they have on suppression efficiency. We return to this difference between the ranges of effects detected by these two assays in Discussion.

One tRNA, U37, has an especially high FR, which suggests that this position may be important for frame maintenance from the $\mathrm{E}$ site. Not all of the position 37 mutants have high FRs. C37 does not have an especially high FR. Unfortunately, G38 is inactive in our assay. It was also almost inactive in the Yarus et al. (1986) study. They suggested that this mutation might create a C:G base pair across the bottom of the anticodon stem, which would severely impact the structure of the anticodon loop, and thus cause very low translational activity. To study the effect of the G38 mutation in a tRNA that does not have this base pair, we assayed a U32-G38 double mutant. The secondary $\mathrm{C}>\mathrm{U} 32$ mutation may at least partially disrupt the potential stem-lengthening base pair. Yarus et al. (1986) found that the double mutant has significant suppression efficiency, and we observed substantial $\beta$-galactosidase activities for both the shift and no-shift reporters (Table 1). Thus, the U32 secondary mutation partially restores translational function to the G38 mutant. However, its FR is within the range exhibited by other Su7 mutants that have similar suppression efficiencies (Fig. 3), which suggests that nucleotide G38 does not make Su7 especially shiftprone from the $\mathrm{E}$ site. Altogether, these results suggest that the high FR for U37 is due to some property specific to uridine rather than to the loss of the WT nucleotide.

\section{The Hirsh mutation increases FR}

The G>A24 mutation (the Hirsh mutation), which occurs in the base-paired stem of the dihydrouridine stem-loop, has been observed to increase A site selection of tRNA ${ }^{\text {Trp }}$ and its variants that contain it. For example, it causes tRNA $^{\text {Trp }}$ to suppress UGA nonsense mutations despite a A:C mispair at the third codon position (Hirsh 1971; Hirsh and Gold 1971), and it increases the Su7 suppression efficiencies at both UAG and UUA nonsense mutants (Raftery et al. 1986). Suppression of the latter codon requires an A:C mispair. Moreover, this mutation, as well as others in this region of the tRNA, increase first-position misreading by Su7 derivatives (Schultz and Yarus 1994a, b). Yarus and Smith (1995) suggested that the Hirsh mutation alters the flexibility of the tRNA, allowing it to more readily adopt confirmations necessary for selection in the A site. Recent data by Cochella and Green (2005) shows that the mutation substantially increases the forward rate constants for aminoacyl-tRNA selection at near cognate codons, which is consistent with this model.

The Hirsh mutation causes a $\sim 25 \%$ decrease in no-shift activity and a $\sim 15 \%$ increase in shift activity. Two conclusions emerge from these changes in activities. One conclusion is that FR increases by about $50 \%$ relative to Su7. We suggest that this increased conformational adaptability for the A site may come at the cost of decreased $\mathrm{E}$ site affinity. It is possible that tRNA conformational changes important for acceptance in the A site are also important for tRNA dissociation from the $\mathrm{E}$ site.

The other conclusion is that the combined shift plus noshift activity is reduced by the mutation, which is unexpected because the Hirsh mutation generally increases translational activity when estimated from suppression efficiencies. The reason for this apparent decrease in overall translational activity in our system is not clear. It is possible that the mutation decreases the ability of Su7 to read the amber codon in this specific context.

\section{Loss of the $\mathrm{ms}^{2} \mathrm{io}^{6} \mathrm{~A}$ modification at $\mathrm{A} 37$ does not increase frame loss from the $\mathrm{E}$ site}

The results above suggest that weak suppressor tRNAs may be less able to help hold the reading frame while in the $\mathrm{E}$ site, with one very weak suppressor (U37) being especially poor at this task. All tRNAs that read codons that begin with $U$ have a hypermodified A37. In E. coli, the modified nucleotide is $\mathrm{ms}^{2} \mathrm{i}^{6} \mathrm{~A} 37$ (2-methylthio-N-6-isopentenyl adenosine 37), while in Salmonella, such tRNAs have the similar $\mathrm{ms}^{2}$ io $^{6} \mathrm{~A} 37$ nucleotide in which the isopentenyl group is cis-hydroxylated (reviewed in Björk 1995). In Salmonella, the miaA1 mutation blocks all modification of A37 (Ericson and Björk 1986). The miaAl mutation decreases polypeptide elongation rate (Ericson and Björk 1986), and also decreases the suppression efficiencies of 
suppressor tRNAs that normally carry these modifications, although the effects vary depending on the tRNA and the context of the suppressed nonsense codon (Bouadloun et al. 1986). Finally, miaA1 also decreases the apparent rate of aminoacyl-tRNA selection on the ribosome ( $\mathrm{Li}$ et al. 1997).

To determine whether the loss of the hypermodification at position 37 also increases FR, we performed assays with the WT Su7 in miaAt and miaA1 hosts (Table 2). In the miaA+ WT Salmonella host, $\beta$-galactosidase activities are considerably lower than those in E. coli. Moreover, FR in Salmonella is about five times greater than in E. coli. These differences may be due to a reduction in Su7 performance in Salmonella that affects both its ability to translate (lower activities) and to hold the reading frame (higher FR). Reasons for these differences between these closely related bacterial species are not obvious.

The miaA1 mutation decreases $\beta$-galactosidase activities by about $50 \%$. This decrease is consistent with a reduced ability of the unmodified tRNA to suppress the UAG codon when it appears in the A site, and agrees with observations that the miaA1 mutation decreases the suppression efficiencies of other suppressors (Bouadloun et al. 1986). Importantly for this assay, such a decrease should equally affect both the shift and no-shift reporters because suppression of the codon in the A site is prerequisite to either activity. The miaA1 mutation also causes a $\sim 25 \%$ decrease in FR. These results are not consistent with the hypothesis that the $\mathrm{ms}^{2}$ io $^{6} \mathrm{~A} 37$ modifications are important to the ability of Su7 to help hold the reading frame from the $\mathrm{E}$ site.

To determine whether the miaAl mutation increases frame loss by tRNAs that read sense codons might also increase frameshifting, we performed assays with sense codons in the E site position. We analyzed six lacZ/RF2 alleles that have different sense codons at the $\mathrm{E}$ site position in an miaA1 Salmonella strain. Three of the lacZ-RF2 alleles have $\mathrm{E}$ site codons that are read by $\mathrm{ms}^{2}{ }^{2}{ }^{6} \mathrm{~A}$ containing tRNA and, as controls, the other three are read by tRNAs that contain $\mathrm{m}^{1} \mathrm{G}$ instead (1-methyl-guanosine). The latter three constructs control for general effects of the miaAl mutation on the $\beta$-galactosidase activities of RF2:lac $Z$ reporters. The codons in the $\mathrm{A}$ and $\mathrm{P}$ sites at the time of the frameshift are read by tRNAs that do not contain either $\mathrm{ms}^{2}$ io $^{6} \mathrm{~A} 37$ or $\mathrm{m}^{1} \mathrm{G}$ (see Materials and Methods).

We measured $\beta$-galactosidase activities in isogenic miaAl and miaA $^{+}$strains and calculated miaAl-/mia+ ratios of those activities. The results are shown in Table 3. If the absence of the $\mathrm{ms}^{2}{ }^{2} \mathrm{io}^{6} \mathrm{~A} 37$ modifications cause the tRNA to be more frameshift-prone from the $\mathrm{E}$ site, then the miaA1-/+ ratio should be higher for alleles read by A37 tRNAs. However, the miaA1-/mia + ratios of the three alleles read by such tRNAs are statistically indistinguishable from those of the alleles read by tRNAs with $\mathrm{m}^{1} \mathrm{G} 37$. These data show that the absence of the $\mathrm{ms}^{2}$ io ${ }^{6} \mathrm{~A} 37$ modifications does not appreciably alter the framing properties of these sense tRNAs while they are in the E site.

\section{DISCUSSION}

We have measured the effects of tRNA anticodon stemloop mutations on the ability of tRNA to help hold the reading frame while in the $\mathrm{E}$ site. There are four significant observations. First, for a set of mutant amber suppressor tRNAs, there is a weak inverse correlation between suppression efficiency and increased frameshifting from the E site. Second, one particularly weak suppressor, Su7-U37, is especially frameshift prone. Third, the D stem "Hirsh" mutation, which has been shown to increase misreading in the ribosomal $\mathrm{A}$ site by aminoacyl-tRNA ${ }^{\operatorname{Trp}}$ and its derivatives, increases frame loss when tRNA is in the E site. Fourth, the miaA1 mutation, which eliminates the $\mathrm{ms}^{2}{ }^{2}{ }^{6} \mathrm{~A}$ nucleotide hypermodification of tRNAs that read codons starting with $U$, does not decrease the ability of tRNA to help hold the reading frame from the E site.

Previously, we showed (Curran and Yarus 1986) that these anticodon stem-loop mutations do not affect reading frame maintenance in a different assay. In that work, we tested the idea that altered anticodon stem-loop sequences might perturb translocation. Because we were not looking specifically for effects from the E site, we did not place the suppressible UAG codon immediately upstream of a slippery site and, therefore, we would not have detected frameshifting resulting from $\mathrm{P}$ site slippage from that codon. In addition, the UAG was located deep into the lac $Z$ coding sequence where conservative substitutions at the $3^{\prime}$ neighboring codon have measurable reductions in lac $Z$ activity (Curran and Yarus 1987); therefore, our constructs might not have been able to report ribosomal hopping because any consequent polypeptides probably would not have been active. Thus, although the current assay can reliably detect

TABLE 2. Effects of the miaA1 mutation on FR by Su7

\begin{tabular}{lccccc}
\hline $\begin{array}{l}\text { Shift activity } \\
\text { in miaA+ host }\end{array}$ & $\begin{array}{c}\text { No-shift activity } \\
\text { in miaA+ host }\end{array}$ & $\begin{array}{c}\text { FR } \\
\text { in miaA+ host }\end{array}$ & $\begin{array}{c}\text { Shift activity } \\
\text { in miaA1 host }\end{array}$ & $\begin{array}{c}\text { No-shift activity } \\
\text { in miaA1 host }\end{array}$ & $\begin{array}{c}\text { FR } \\
\text { in miaA1 host }\end{array}$ \\
\hline $830 \pm 30$ & $1480 \pm 65$ & $0.56 \pm 0.04$ & $310 \pm 20$ & $740 \pm 25$ & $0.42 \pm 0.04$ \\
\hline $\begin{array}{l}\text { Strains all carry the Su7-endoding plasmid and either the shift or no-shift reporter. The lacZ reporters are outlined in Figure 2. Activities are } \\
\beta \text {-galactosidase activities } \pm \text { standard errors of the mean. FR is the shift/no-shift ratio. }\end{array}$
\end{tabular}


TABLE 3. $\beta-$ Galactosidase activities of RF2- lacZ alleles that have different codons in the E site

\begin{tabular}{lccr}
\hline $\begin{array}{l}\text { E site codon (tRNA } \\
\text { position } 37 \text { nucleotide) }\end{array}$ & $\begin{array}{c}\beta \text {-Galactosidase } \\
\text { activity in the } \\
\text { miaA1 strain }\end{array}$ & $\begin{array}{c}\beta \text {-Galactosidase } \\
\text { activity in the } \\
\text { miaA+ strain }\end{array}$ & miaA1/miaA+ \\
\hline UAU (ms $\left.{ }^{2} \mathrm{io}^{6} \mathrm{~A} 37\right)$ & $3230 \pm 230$ & $3580 \pm 360$ & $0.9 \pm 0.17$ \\
$\mathrm{UCU}\left(\mathrm{ms}^{2} \mathrm{io}^{6} \mathrm{~A} 37\right)$ & $3400 \pm 80$ & $3530 \pm 120$ & $1.0 \pm 0.02$ \\
$\mathrm{UGU}\left(\mathrm{ms}^{2} \mathrm{io}^{6} \mathrm{~A} 37\right)$ & $2760 \pm 160$ & $3250 \pm 200$ & $0.85 \pm 0.11$ \\
$\mathrm{CAU}\left(\mathrm{m}^{1} \mathrm{G} 37\right)$ & $1050 \pm 25$ & $1160 \pm 25$ & $0.9 \pm 0.04$ \\
$\mathrm{CCU}\left(\mathrm{m}^{1} \mathrm{G} 37\right)$ & $1640 \pm 130$ & $1480 \pm 50$ & $1.1 \pm 0.14$ \\
$\mathrm{CGU}\left(\mathrm{m}^{1} \mathrm{G} 37\right)$ & $860 \pm 80$ & $830 \pm 70$ & $1.0 \pm 0.23$ \\
\hline
\end{tabular}

The codons are read by tRNAs that have the indicated nucleotide at position 37 . The miaA1 mutation eliminates the $\mathrm{ms}^{2}$ io $^{6} \mathrm{~A} 37$ modifications but should not affect tRNAs with $\mathrm{m}^{1} \mathrm{G} 37$. The RF2 portion constructs contain these sequences: S/D XXU AAA AGG, where S/D is the Shine/Dalgarno-like element and $X X U$ is the variable $E$ site codon. The $P$ and $A$ site codons (AAA and AGG) are decoded by tRNAs that do not contain either $\mathrm{ms}^{2}$ io ${ }^{6} \mathrm{~A} 37$ or $\mathrm{m}^{1} \mathrm{G} 37$. rejected and may have been selected, therefore, to be exquisitely sensitive to anticodon loop structure. It is likely that the A site exploits abnormal anticodon loop features to either increase the offrates and/or decrease the forward rates for aminoacyl-tRNA adaptation with the ribosome (Wintermeyer et al. 2004; Rodnina et al. 2005). Moreover, by amplifying such effects through two independent steps, proofreading further increases sensitivity (Ninio 2006). In contrast, the $\mathrm{E}$ site has presumably not been selected to provide the same high level of discrimination among duplexes and, therefore, may not be nearly as sensitive to anticodon loop microstructure as is the A site.

frame loss by this mutant tRNA set, the previous assay was probably incapable of it.

Although we study the $\mathrm{E}$ site in the context of a programmed frameshift site, other work suggests that a role for E site tRNA in holding the reading frame may be general. This idea was initially suggested by Nierhaus (Blaha and Nierhaus 2001; Nierhaus 2006), and theoretical work suggests that the E site duplex is necessary to hold the frame after translocation until the A site is occupied (Lim and Curran 2001; Lim et al. 2005). The main argument is that the P site duplex alone may not be able to reliably hold the frame because $\mathrm{P}$ site duplex dissociation might occur at rates comparable to those of translocation and aminoacyltRNA selection. If true, then an E site duplex may provide important insurance against frame loss. From that view, the effect of the E site duplex on the RF2 frameshift is its contribution to the kinetic barrier that must be overcome to achieve the programmed frameshift. The same barrier should also apply to frame maintenance at normal sites.

Thus, an altered anticodon loop may reduce the kinetic barrier to removing the E site tRNA. In our previous study (Sanders and Curran 2007), FR was inversely correlated with estimated E site duplex stability. This relationship was studied using two different methods for estimating duplex stability, and the correlations observed for the methods had similar coefficients of determination $\left(r^{2}\right)$ of about 0.5 . These results suggest that about half of the effect on FR can be attributed to differences in duplex stability. The remainder has an unknown cause(s). Therefore, it is likely that at least some of the increases in FR observed for anticodon loop mutants are due to destabilized duplexes.

However, the effects of anticodon loop mutations on FR are generally small compared to the effects that some of these mutations have on suppression efficiency. This observation no doubt results at least in part from the differences in the functions of the $\mathrm{A}$ and $\mathrm{E}$ sites. The $\mathrm{A}$ site is responsible for ensuring that incorrect complexes are
Other evidence suggests that the $\mathrm{E}$ site is less restrictive than the P site. Mutational analyses of the "tandem slippage" programmed frameshift sites show that the upstream codon is generally more tolerant of mismatches that the downstream codon (Jacks et al. 1988; Brierley et al. 1992). Assuming that the two codons are in the $\mathrm{E}$ and $\mathrm{P}$ sites during frameshifting, then the $\mathrm{E}$ site is less sensitive to duplex structure than is the $\mathrm{P}$ site. Thus, the $\mathrm{E}$ site appears to be less sensitive to duplex structure than both of the other coding sites.

From another view, however, mutations that strongly decrease suppression efficiency almost always increase FR. Importantly, the WT anticodon stem-loop has the highest suppression efficiency and the lowest FR. This suggests that least) have all been co-optimized.

One mutant tRNA, U37, has an especially high FR, suggesting that it is unusually frameshift-prone when in the E site. U37 is the weakest suppressor that has measurable activity in our assay (Table 1), and we suggest that the framing defect and the very low suppressor efficiency are related. Position 37 is adjacent to the anticodon, and is strongly correlated to the identity of a neighboring anticodon nucleotide at position 36 (Yarus 1982). Because other mutations at position 37 do not have high FR, this problem is not strictly caused by the loss of the WT nucleotide (2methylthio 6-isopentenyl adenosine $\left.37, \mathrm{~ms}^{2} \mathrm{i}^{6} \mathrm{~A} 37\right)$. Instead, it is more likely due to the chemical nature of uridine at position 37 (U37 occurs naturally only in some mitochondrial tRNAs) (Sprinzl and Vassilenko 2005). There is abundant evidence that position 37 is important for anticodon loop structure and function. Nucleotide 37 is thought to preposition the anticodon for interaction with the codon (reviewed by Agris et al. 2007), and bulky modified nucleotides are often used there, presumably to facilitate stacking interactions (Björk 1995; Curran 1998; Agris et al. 2007). It is possible that U37 simply cannot perform that role. From that view, it is at least plausible that this tRNA forms an especially anticodon stem-loop sequences and the $\mathrm{A}$ and $\mathrm{E}$ sites (at 
weak duplex that accounts for both a high rate of rejection from the A site and a rapid dissociation from the $\mathrm{E}$ site.

$\mathrm{X}$-ray crystallographic studies do not yet give clear results for the structure of the E site codon:tRNA duplex. Recent work by Jenner et al. (2007) shows that the E site codon in post-initiation and elongation ribosomal complexes is in the A-form, which is a prerequisite for pairing with a cognate anticodon. These results differ from those of Selmer et al. (2006), who observe that the E site codon and anticodon are positioned close enough for any interaction with each other. Selmer et al. used a noncognate tRNA so that normal pairing could not occur; but the point is that the codon and anticodon were not in proximity. Nierhaus's (Marquez et al. 2004) and our (Sanders and Curran 2007) functional data suggest that the E site does indeed contain translationally relevant, basepaired duplexes. In fact, our previous data shows that wobbletype base pairs at the third codon position are associated with increased frameshifting, which suggests that base pairing at this position is important for reading frame maintenance (Sanders and Curran 2007). We suggest that the Jenner et al. structure is more closely relevant to the $\mathrm{E}$ site's framing role, although the anticodon in that structure is distorted such that it might not be paired to the third codon position. We predict that there is a functionally important state in which the full codon:anticodon duplex is present in the E site.

In summary, anticodon loop sequence is important for helping to hold the reading frame when tRNA is in the E site. In our assay, at least, the effects are considerably smaller than effects on function in the A site. The E site may also be less restrictive than the $\mathrm{P}$ site. These differences are likely due to differences in the functions of the coding sites.

\section{MATERIALS AND METHODS}

The tRNA-encoding plasmids were the generous gifts of Dr. Mike Yarus, University of Colorado Boulder, and are described in Yarus et al. (1986). They confer tetracycline resistance, and have the pMB9 origin of replication. They are compatible with the lacZRF2 reporter plasmids, which confer chloramphenicol resistance and have the P15A origin. The lacZ-RF2 reporter plasmids were constructed starting with pJC27 (Curran and Yarus 1986), which encodes a pseudo-WT lac $Z$ allele that contains a polylinker near the $5^{\prime}$ end. All cloning methods were performed using strain MY600 (Curran and Yarus 1986). Plasmid derivatives were made by replacing a 20-nucleotide section (between HindIII and BamHI sites) of the polylinker with synthetic oligonucleotides encoding the RF2 frameshift site and having TAG as the E site codon. The shift construct was made using the oligonucleotide below and its complement (the TAG codon is underlined):

\section{AGCTTCCTTAGGGGGTAGCTTCGGCTCTGG}

The no-shift construct was made using oligonucleotides containing one less nucleotide (missing the last " $G$ ").
The Salmonella strains GT522 and GT523 are prototrophic and miaA+ and miaA1, respectively (Ericson and Björk 1986). They were the generous gifts of Dr. Glenn Björk, University of Umeå, Sweden. Experimental lacZ-RF2 frameshift reporter plasmids were constructed as above using oligonucleotides based on the one below. The underlined XXT corresponds to the $\mathrm{E}$ site position during RF2 frameshifting. The AAA AGG codons corresponding to the $\mathrm{P}$ and $\mathrm{A}$ site positions are read by tRNAs that are not affected by the miaA1 mutation. They were introduced into Salmonella via transduction using standard methods (Davis et al. 1980):

\section{AGCTTCCTTAGGGGGXXTAAAAGGCTCTGG}

$\beta$-Galactosidase assays were performed as described in Curran and Yarus (1986).

\section{ACKNOWLEDGMENTS}

We are grateful to Drs. Mike Yarus and Glenn Björk for generous gifts of strains. We also thank Drs. Glenn Björk and Kurt Fredrick for helpful suggestions and comments on the manuscript. This work was supported by NIH Grant GM-077194 to J.F.C.

Received May 6, 2008; accepted June 9, 2008.

\section{REFERENCES}

Agris, P.F., Vendeix, F.A.P., and Graham, W.D. 2007. tRNA's wobble decoding of the genome: 40 years of modification. J. Mol. Biol. 366: $1-13$.

Baranov, P.V., Gesteland, R.F., and Atkins, J.F. 2004. P-site tRNA is a crucial initiator of ribosomal frameshifting. RNA 10: 221-230.

Björk, G.R. 1995. Biosynthesis and function of modified nucleosides. In tRNA: Structure, biosynthesis and function (eds. D. Söll and U. RajBhandary), pp. 165-205. ASM Press, Washington, DC.

Björnsson, A. and Isaksson, L.A. 1993. UGA codon context which spans three codons. Reversal by $\mathrm{ms}^{2} \mathrm{i}^{6} \mathrm{~A} 37$ in tRNA, mutation in rpsD(S4) or streptomycin. J. Mol. Biol. 232: 1017-1029.

Blaha, G. and Nierhaus, K.H. 2001. Features and functions of the ribosomal E site. Cold Spring Harb. Symp. Quant. Biol. 66: 135-146.

Bouadloun, F., Srichaiyo, T., Isaksson, L.A., and Björk, G.R. 1986. Influence of modification next to the anticodon in tRNA on codon context sensitivity of translational suppression and accuracy. $J$. Bacteriol. 166: 1022-1027.

Brierley, I., Jenner, A.J., Stephen, C., and Inglis, S.C. 1992. Mutational analysis of the "slippery-sequence" component of a coronavirus ribosomal frameshifting signal. J. Mol. Biol. 227: 463-479.

Cabello-Villegas, J., Winkler, M.E., and Nikonowicz, E.P. 2002. Solution conformations of unmodified and $\mathrm{A}_{37} \mathrm{~N}^{6}$-dimethylallyl modified anticodon stem-loops of Escherichia coli tRNA ${ }^{\text {Phe }}$. J. Mol. Biol. 319: 1015-1034.

Cochella, L. and Green, R. 2005. An active role for tRNA in decoding beyond codon:anticodon pairing. Science 308: 1178-1180.

Curran, J.F. 1993. Analysis of effects of tRNA:message stability on frameshift frequency at the Escherichia coli RF2 programmed frameshift site. Nucleic Acids Res. 21: 1837-1843.

Curran, J.F. 1998. Modified nucleosides in translation. In Modification and editing of RNA: The alteration of RNA structure and function (eds. $\mathrm{H}$. Grosjean and R. Benne), pp. 493-516. ASM Press, Washington, DC.

Curran, J.F. and Yarus, M. 1986. Base substitutions in the tRNA anticodon arm do not degrade the accuracy of reading frame maintenance. Proc. Natl. Acad. Sci. 83: 1638-1642. 
Curran, J.F. and Yarus, M. 1987. Reading frame selection and Transfer RNA anticodon loop stacking. Science 238: 1545-1550.

Curran, J.F. and Yarus, M. 1988. Use of tRNA suppressors to probe regulation of E. coli RF-2. J. Mol. Biol. 203: 75-83.

Curran, J.F. and Yarus, M. 1989. Rates of tRNA selection by 29 specific codons in vivo. J. Mol. Biol. 209: 65-77.

Davis, R.W., Botstein, D., and Roth, J.R. 1980. A manual for genetic engineering: Advanced bacterial genetics. Cold Spring Harbor Laboratory Press, Cold Spring Harbor, NY.

Durant, P.C., Bajji, A.C., Sundaram, M., Kumar, R.K., and Davis, D.R. 2005. Structural effects of hypermodified nucleosides in the Escherichia coli and human tRNA ${ }^{\text {Lys }}$ anticodon loop: The effect of nucleosides $\mathrm{s}^{2} \mathrm{U}, \mathrm{mcm}^{5} \mathrm{U}, \mathrm{mcm}^{5} \mathrm{~s}^{2} \mathrm{U}, \mathrm{mnm}^{5} \mathrm{~s}^{2} \mathrm{U}, \mathrm{t}^{6} \mathrm{~A}$, and $\mathrm{ms}^{2} \mathrm{t}^{6} \mathrm{~A}$. Biochemistry 44: 8078-8089.

Ericson, J.U. and Björk, G.R. 1986. Pleiotropic effects induced by modification deficiency next to the anticodon of tRNA from Salmonella typhimurium LT2. J. Bacteriol. 166: 1013-1021.

Hirsh, D. 1971. Tryptophan tRNA as the UGA suppressor. J. Mol. Biol. 58: $439-468$.

Hirsh, D. and Gold, L. 1971. Translation of the UGA triplet in vitro by tryptophan tRNAs. J. Mol. Biol. 58: 459-468.

Houssier, C. and Grosjean, H. 1985. Temperature jump relaxation studies on the interactions between transfer RNAs with complementary anticodons. The effect of modified bases adjacent to the anticodon triplet. J. Biomol. Struct. Dyn. 3: 387-408.

Jacks, T., Madhani, H.D., Masiarz, F.R., and Varmus, H.E. 1988. Signals for ribosomal frameshifting in the Rous sarcoma virus gagpol region. Cell 55: 447-458.

Jenner, L., Rees, B., Yusupov, M., and Yusupova, G. 2007. Messenger RNA conformations in the ribosomal $\mathrm{E}$ site revealed by X-ray crystallography. EMBO Rep. 8: 846-850.

Li, J.-n., Esberg, B., Curran, J.F., and Björk, G.R. 1997. Three modified nucleosides present in the anticodon stem and loop influence the in vivo aa-tRNA selection in a tRNA-dependent manner. J. Mol. Biol. 271: 209-221.

Liao, P.-Y., Gupta, P., Petrov, A.N., Dinman, J.D., and Le, K.H. 2008. A new kinetic model reveals the synergistic effect of E-, P-, and A-sites on +1 ribosomal frameshifting. Nucleic Acids. Res. 36: 26192629.

Lim, V.I. and Curran, J.F. 2001. Analysis of codon:anticodon interactions within the ribosome provides new insights into codon reading and the genetic code structure. RNA 7: 942-957.

Lim, V.I., Curran, J.F., and Garber, M. 2005. Ribosomal elongation cycle: Energetic, kinetic and stereochemical aspects. J. Mol. Biol. 351: $470-480$.

Marquez, V., Wilson, D.N., Tate, W.P., Triana-Alonso, F., and Nierhaus, K.H. 2004. Maintaining the translational reading frame: The influence of the $\mathrm{E}$ site during translational regulation of release factor 2. Cell 118: 45-55.

Nierhaus, K.H. 2006. Decoding errors and the involvement of the E-site. Biochimie 88: 1013-1019.

Ninio, J. 2006. Multiple stages in codon-anticodon recognition: Double-trigger mechanisms and geometric constraints. Biochimie 88: $963-992$.
Raftery, L.A. and Yarus, M. 1987. Systematic alterations in the anticodon arm make tRNA ${ }^{\mathrm{Glu}}{ }_{-} \mathrm{Su}_{\mathrm{oc}}$ a more efficient suppressor. EMBO J. 6: 1499-1506.

Raftery, L.A., Bermingham Jr., J.R., and Yarus, M. 1986. Mutation in the D arm enables a suppressor with a CUA anticodon to read both amber and ochre codons in Escherichia coli. J. Mol. Biol. 190: 513-517.

Rodnina, M.V., Gromadski, K.B., Kothe, U., and Wieden, H.J. 2005. Recognition and selection of tRNA in translation. FEBS Lett. 579: 938-942.

Sanders, C.L. and Curran, J.F. 2007. Genetic analysis of the E site during RF2 programmed frameshifting. RNA 13: 1483-1491.

Schultz, D.W. and Yarus, M. 1994a. tRNA structure and ribosomal function. I. tRNA nucleotide 27-43 mutations enhance first position wobble. J. Mol. Biol. 235: 1381-1394.

Schultz, D.W. and Yarus, M. 1994b. tRNA structure and ribosomal function. II. Interaction between anticodon helix and other tRNA mutations. J. Mol. Biol. 235: 1395-1405.

Selmer, M., Dunham, C.M., Murphy, F.V., Weixlbaumer, A., Petry, S., Kelley, A.C., Weir, J.R., and Ramakrishnan, V. 2006. Structure of the 70S ribosome complexed with mRNA and tRNA. Science 313: 1935-1942.

Sergiev, P.V., Lesnyalk, D.V., Kiparisov, S.V., Burakovsky, D., Leonov, L., Bogdanov, A.A., Brimacombe, R., and Dontsova, O. 2005. Function of ribosomal E-site: A mutagenesis study. Nucleic Acids Res. 33: 6048-6056.

Sprinzl, M. and Vassilenko, K.S. 2005. Compilation of tRNA sequences and sequences of tRNA genes. Nucleic Acids Res. 139: D139D140.

Stuart, J.W., Koshlap, K.M., Guenther, R., and Agris, P.F. 2003. Naturally-occurring modification restricts the anticodon domain conformational space of tRNA ${ }^{\text {Phe }}$.J. Mol. Biol. 334: 901-918.

Weiss, R.B., Dunn, D.M., Dahlberg, A.E., Atkins, J.F., and Gesteland, R.F. 1988. Reading frame switch caused by base-pair formation between the 3 ' end of 16S rRNA and the mRNA during elongation in protein synthesis in Escherichia coli. EMBO J. 7: 1503-1507.

Weiss, R.B., Dunn, D.M., Atkins, J.F., and Gesteland, R.F. 1990. Ribosomal frameshifting from -2 to +50 nucleotides. Prog. Nucleic Acids Res. Mol. Biol. 39: 159-183.

Wintermeyer, W., Peske, F., Beringer, M., Gromadski, K.B., Savalsbergh, A., and Rodnina, M.V. 2004. Mechanisms of elongation on the ribosome: Dynamics of a macromolecular machine. Biochem. Soc. Trans. 32: 733-737.

Yarus, M. 1982. Translational efficiency of Transfer RNA: Uses of an extended anticodon. Science 218: 646-652.

Yarus, M. and Smith, D. 1995. tRNA on the ribosome: A waggle theory. In $t R N A$ : Structure, biosynthesis and function (eds. D. Söll and U. RajBhandary), pp. 443-469. ASM Press, Washington, DC.

Yarus, M., Cline, S.W., Wier, P., Breeden, L., and Thompson, R.C. 1986. Actions of the anticodon arm in translation: On the phenotypes of tRNA mutants. J. Mol. Biol. 192: 235-255. 

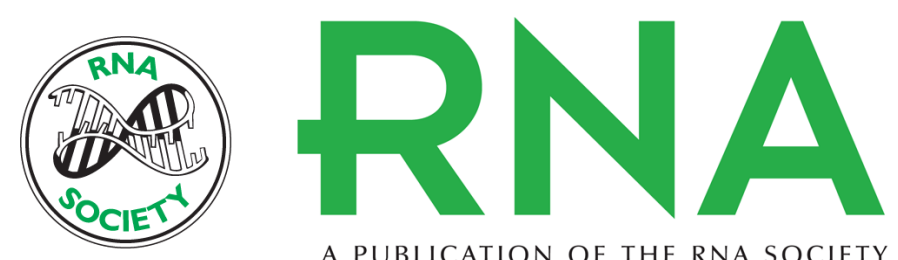

A PUBLICATION OF THE RNA SOCIETY

\section{Anticodon loop mutations perturb reading frame maintenance by the $E$ site tRNA}

Christina L. Sanders, Kristin J. Lohr, Holly L. Gambill, et al.

RNA 2008 14: 1874-1881 originally published online July 30, 2008

Access the most recent version at doi:10.1261/rna.1170008

References This article cites 42 articles, 9 of which can be accessed free at:

http://rnajournal.cshlp.org/content/14/9/1874.full.html\#ref-list-1

\section{License} Email Alerting
Service $\begin{aligned} & \text { Receive free email alerts when new articles cite this article - sign up in the box at the } \\ & \text { top right corner of the article or click here. }\end{aligned}$ 